\title{
Thermal properties of compressed expanded graphite: photothermal measurements
}

\author{
J. Bodzenta $\cdot$ J. Mazur · A. Kaźmierczak-Bałata
}

Received: 23 October 2010 / Revised version: 29 March 2011 / Published online: 30 April 2011

(C) The Author(s) 2011. This article is published with open access at Springerlink.com

\begin{abstract}
The thermal diffusivity and the thermal conductivity of compressed expanded graphite (CEG) samples were investigated by photothermal measurements in two geometries differing by a place of temperature disturbance detection. This disturbance can be detected on a surface opposite to the one at which the disturbance was generated (rear detection) or on the same surface (front detection). A measurement based on the rear detection allowed us to determine the effective thermal diffusivity of the sample, while the method with front detection gives the possibility of analysis of homogeneity of the sample. It is shown that the thermal diffusivity of CEG strongly depends on its apparent density. Moreover, CEG samples reveal anisotropy of the thermal properties. The thermal diffusivity in the direction parallel to the compacting axis is lower than the one in the direction perpendicular to it. The parallel thermal diffusivity decreases with growing apparent density, while the perpendicular thermal diffusivity significantly grows when the apparent density grows. The perpendicular thermal conductivity exhibits the same behavior as the perpendicular thermal diffusivity. The parallel thermal conductivity slightly grows with growing density and then reaches a plateau. The anisotropy of CEG samples grows with growing apparent density and vanishes for low-density samples. The photothermal measurement with front signal detection revealed that the CEG samples are non-homogeneous in the direction of the compacting axis and can be modeled by a two-layer system.
\end{abstract}

J. Bodzenta ( $\varangle)$ · J. Mazur · A. Kaźmierczak-Bałata Institute of Physics, Silesian University of Technology, Krzywoustego 2, 44-100 Gliwice, Poland

e-mail: jerzy.bodzenta@polsl.pl

\section{Introduction}

Unique properties of different carbon allotropes cause carbon materials to have found numerous applications. Diamond, an excellent electrical insulator with the highest thermal conductivity, is also known for extremely high hardness and remarkable optical properties. The mentioned properties make diamond attractive for cutting and abrasive tools, substrates for high-power electronic devices, and windows for high-power optics. The other carbon allotrope, graphite, chemically more stable than diamond, is an electrical conductor. Many applications of graphite are connected with its self-lubricated properties, but it is also used as a refractory material, a neutron moderator in nuclear reactors, and a material for electrodes. Besides these 'classical' forms of carbon, there is continuous development of new carbon materials based on molecules built from carbon atoms, e.g. fullerenes.

In graphite, carbon atoms create regular hexagons organized in plane sheets-graphene. The graphene structure is held by strong covalent bonds. Relatively weak van der Waals forces bond graphene layers to form the crystal structure of the graphite. The character of van der Waals bonds allows the insertion of various atoms and molecules between graphene layers-intercalation of the graphite [1]. A representative review of the preparation, the properties, and the applications of the intercalated graphite can be found elsewhere [2]. For this work, it is important that the intercalation is the first step to obtain expanded (or exfoliated) graphite. The exfoliation process and basic properties of expanded graphite are described in [3]. During an exfoliation, a rapid vaporization of intercalate destroys van der Waals bonds and leads to expansion of the graphite in the direction of the $c$ axis (perpendicular to graphene planes) up to hundreds of times. Natural graphite flakes convert into long 'worms' 
with honeycomb microstructure. The reported density of the 'worms' is about $15 \mathrm{~kg} \mathrm{~m}^{-3}$ and their porosity exceeds $99 \%$ [4]. For a comparison, the density of non-porous graphite is $2250 \mathrm{~kg} \mathrm{~m}^{-3}$ [5]. Expanded graphite is commercially available in the form of a powder with the apparent density of a few $\mathrm{kg} \mathrm{m}^{-3}$.

Applications of expanded graphite are mainly connected with its chemical stability and possibility of compaction to form final products with controlled thermal conductivity. Raw expanded graphite is used in metallurgical industries to cover molten metal. In this case, it creates thermal insulation and protects against oxidation. Foils produced from expanded graphite are used for gaskets, heat sinks, and packages. Many examples of possible expanded graphite applications can be found in WWW pages of companies offering graphite products.

As is mentioned above, compacted expanded graphite (CEG) is often used for heat management. An important property of CEG is that its thermal conductivity and thermal diffusivity depend on the compaction ratio, i.e. the apparent density of a sample. This behavior has been examined in a few papers [5-7]. Bonnissel et al. determined the thermal diffusivity and the thermal conductivity of CEG samples with the apparent density varying from 20 to $1800 \mathrm{~kg} \mathrm{~m}^{-3}$ [5]. Measurements were carried out for directions parallel and perpendicular relatively to the direction of compression. The thermal diffusivity in the parallel direction decreases when the apparent density of the sample increases. Quite the opposite, when the sample density grows the thermal diffusivity in the perpendicular direction grows. The perpendicular thermal diffusivity is higher than the parallel one and for dense samples is higher than the thermal diffusivity of metals. The parallel thermal conductivity reaches a maximum for samples with the density of about $300 \mathrm{~kg} \mathrm{~m}^{-3}$ and slightly drops for more dense samples, while the one in the perpendicular direction monotonically grows with growing apparent density. The perpendicular thermal conductivity is about two orders of magnitude higher than the parallel one and reaches a value of $500 \mathrm{~W} \mathrm{~m}^{-1} \mathrm{~K}^{-1}$ for very dense samples. Vovchenko et al. measured the thermal conductivity and the thermal diffusivity of CEG in the parallel direction with the apparent density of (330-1950) $\mathrm{kg} \mathrm{m}^{-3}$ [6]. They observed a decrease of the thermal diffusivity with growing density and a minimum of the thermal conductivity at $1500 \mathrm{~kg} \mathrm{~m}^{-3}$. Afanasov et al. revealed that the thermal conductivity of CEG in the parallel direction depends on the parameters of the exfoliation process [7]. Similarly to the results obtained by Bonnissel et al., they also observed growth of the parallel thermal conductivity with growing apparent density for densities less than $200 \mathrm{~kg} \mathrm{~m}^{-3}$ and a plateau for more dense samples. The aim of this work is to investigate the thermal diffusivity and the thermal conductivity of CEG samples of relatively low density. An analysis of the dependence of the anisotropy of thermal properties on the apparent density was also carried out. Additional measurements were done to check the homogeneity of CEG samples in the parallel direction.

\section{Experiment}

\subsection{Samples}

Samples were prepared from EG powder of an apparent density of $11 \mathrm{~kg} \mathrm{~m}^{-3}$ provided by SGL Carbon (Germany) by compression of raw powder in a long tube with square cross section. The inner cross section of the tube was $4 \mathrm{~cm}^{2}$. After weighing, portions of EG powder were compacted to obtain the required density. The apparent density of the samples was in the range (45-241) $\mathrm{kg} \mathrm{m}^{-3}$. The samples were cuboids with square cross section of 2-cm side and 1-2-cm height. Table 1 contains basic characteristics of all investigated samples.

To analyze the anisotropy of the thermal properties of the CEG, 1-cm-thick plates with walls parallel to the compressing direction were cut out of selected samples. Before cutting, a sample was clamped in a special holder which ensured the obtaining a sample of the desired thickness and with parallel walls. A thin steel wire was used to cut the sample. This technique allows us to prepare samples with flat, smooth surfaces. The same method was also used to cut samples for an analysis of their homogeneity.

Table 1 Basic characteristics of investigated samples. The porosity was calculated in relation to non-porous graphite (density $2250 \mathrm{~kg} \mathrm{~m}^{-3}$ )

\begin{tabular}{llllcl}
\hline & $\begin{array}{l}\text { Mass } \\
{[\mathrm{g}]}\end{array}$ & $\begin{array}{l}\text { Thickness } \\
{[\mathrm{cm}]}\end{array}$ & $\begin{array}{l}\text { Volume } \\
{\left[\mathrm{cm}^{3}\right]}\end{array}$ & $\begin{array}{l}\text { Apparent density } \\
{\left[\mathrm{kg} \mathrm{m}^{-3}\right]}\end{array}$ & $\begin{array}{l}\text { Porosity } \\
(\%)\end{array}$ \\
\hline GEP-05 & 0.323 & 1.08 & 4.98 & 65 & 97.1 \\
GEP-06 & 0.829 & 1.22 & 6.16 & 135 & 94.0 \\
GEP-07 & 0.249 & 1.05 & 4.88 & 51 & 97.7 \\
GEP-08 & 0.905 & 1.27 & 5.94 & 152 & 93.2 \\
GEP-09 & 0.218 & 1.21 & 4.20 & 52 & 97.7 \\
GEP-10 & 0.673 & 1.27 & 5.04 & 134 & 94.1 \\
GEP-11 & 0.362 & 1.26 & 4.82 & 75 & 96.7 \\
GEP-12 & 0.903 & 1.54 & 4.62 & 196 & 91.3 \\
GEP-13 & 0.482 & 1.50 & 5.05 & 95 & 95.8 \\
GEP-14 & 0.194 & 1.16 & 4.28 & 45 & 98.0 \\
GEP-21 & 1.238 & 1.29 & 5.14 & 241 & 89.3 \\
GEP-22 & 1.237 & 1.84 & 7.29 & 170 & 92.5 \\
GEP-23 & 1.328 & 1.43 & 5.69 & 233 & 89.6 \\
\hline
\end{tabular}




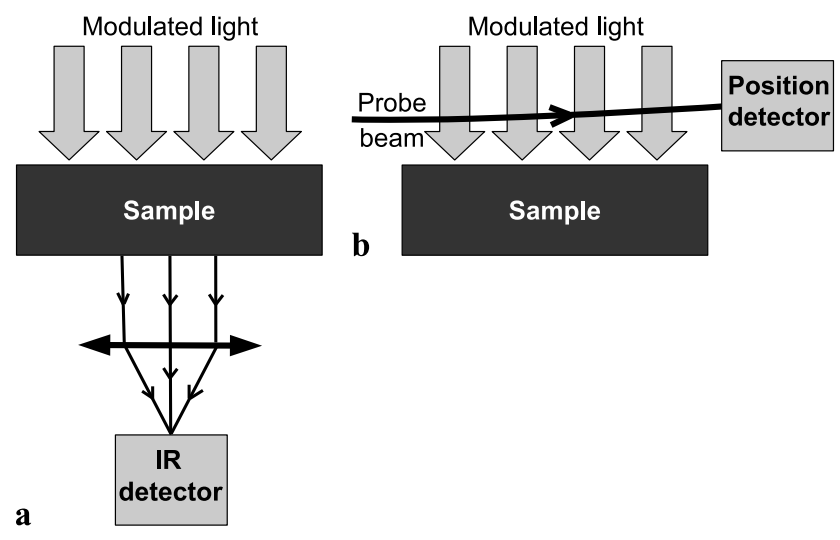

Fig. 1 Geometry of photothermal measurements with rear (a) and front (b) detection of temperature disturbance

\subsection{Thermal measurements}

The thermal diffusivity was measured by continuous-wave photothermal techniques in two geometries shown in Fig. 1. The majority of experiments were carried out in the geometry presented in Fig. 1a. The front sample surface was evenly illuminated by intensity-modulated light from a high-power, white LED with luminous flux of $450 \mathrm{~lm}$ (EPBX-4S00, Edison Opto, Taiwan). Because of a high divergence of the light from this LED and difficulties of its focusing by standard optics, the diode was placed close to the investigated sample and additional focusing is not applied. The temperature disturbance caused by absorption of the light in the sample was detected by infrared (IR) radiometry. The IR radiation from the rear surface of the sample was collected by a germanium lens and measured by an IR detector (PVI2TE-5, VIGO, Poland). Unfortunately, a placement of the LED makes radiometric measurement of the temperature of the illuminated surface impossible. An output of the detector was connected to a lock-in amplifier (SR-830, Stanford Research, USA) and the amplitude and the phase of the signal component at the modulation frequency were measured. The phase was measured in relation to the intensity of light heating the sample. The determination of the thermal diffusivity was based on the analysis of the phase as a function of the modulation frequency. This method is a continuouswave analogue of the standard flash method [8]. For opaque samples, the phase-lag measurements were provided from transmission of a thermal wave through the sample. In the case of non-homogeneous samples, this technique gives information about an effective (averaged) thermal diffusivity of the samples, but does not allow us to analyze the nonhomogeneity.

The idea of a measurement carried out in the geometry shown in Fig. 1b is different. As in the previous case, the temperature disturbance is generated by modulated light. But, signal detection takes place at or near the illuminated surface. The interference of thermal waves generated at this surface appears and waves reflect from the rear surface and possible inner boundaries. Thus, the signal dependence on the modulation frequency should be sensitive to the inner structure of the sample. It means that measurement carried out in this geometry can be used for investigation of the homogeneity of the sample. In our case, the sample was illuminated by a laser diode (G081PU11500m, Roithner LaserTechnik GmbH, Austria). Its central wavelength is $808 \mathrm{~nm}$ and the maximum $\mathrm{CW}$ output power is $1.5 \mathrm{~W}$. The signal detection was based on the mirage effect. A probe beam from a HeNe laser (LGX 7672, Lasos LaserTechnik $\mathrm{GmbH}$, Germany) passed near the heated surface and sustained the deflection on a temperature gradient in the air above the sample. The deflection of the probe beam was detected by a position detector (DL400-7 PSBA, Silicon Sensor International AG, Germany). The signal from the detector was analyzed by a lock-in amplifier (SR-830, Stanford Research, USA) to determine its amplitude and phase at the particular modulation frequency.

As was mentioned above, experiments carried out using mirage-effect detection were supplementary ones. This is why the authors did not decide to rearrange the experimental setup to use the radiometric temperature detection. Moreover, the measurements carried out prove that experiments performed in both configurations give consistent results.

Basic results used for the analysis of the thermal properties were obtained in the same one-dimensional (1D) geometry. The applied measuring technique leads to averaging of the thermal properties over the sample thickness. Therefore, the obtained results are quite insensitive to local heterogeneities. In a case of photothermal methods using local excitation (point-like source of the thermal wave), local thermal properties are investigated. For highly porous CEG samples, the results of such measurement may considerably depend on a position at the sample surface. So, the 1D method seems to be more suitable to characterize the sample as a whole.

More details about measurements in both geometries can be found elsewhere [9].

\subsection{Signal analysis}

The analysis of measured dependences is based on a 1D model of thermal wave propagation in a layered structure. It was assumed that a sample consists of $N$ layers perpendicular to the $z$ axis and is surrounded by air (Fig. 2). Each layer is described by its thickness $d_{n}$, the thermal diffusivity $\alpha_{n}$, the thermal effusivity $\varepsilon_{n}$, and the density of heat sources $w_{n}(z, t)$. The temperature in the $n$th layer has to fulfill the Fourier-Kirchhoff equation

$$
\frac{\partial^{2} T_{n}}{\partial z^{2}}=\frac{1}{\alpha_{n}} \frac{\partial T_{n}}{\partial t}-\frac{w_{n}(z, t)}{\varepsilon_{n} \sqrt{\alpha_{n}}} .
$$




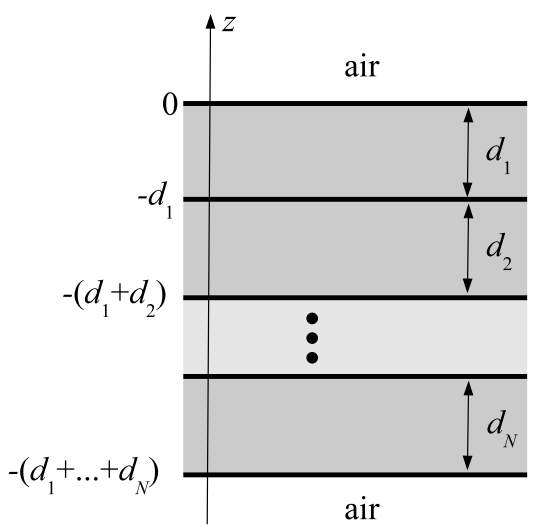

Fig. $2 N$-Layer structure

Assuming the harmonic heat source

$w_{n}(z, t)=w_{0 n}(z) \exp (\mathrm{i} \omega t)$

where $\omega$ is the modulation frequency, (1) can be transformed into the form

$\frac{\partial^{2} \theta_{n}}{\partial z^{2}}=\frac{\mathrm{i} \omega}{\alpha_{n}} \theta_{i}-\frac{w_{0 n}(z)}{\varepsilon_{n} \sqrt{\alpha_{n}}}$,

where $\theta_{i}$ is the harmonic temperature component of frequency $\omega$. The solution of (3) is

$\theta_{n}(z)=A_{n} \exp \left(\sqrt{\frac{\mathrm{i} \omega}{\alpha_{n}} z}\right)+B_{n} \exp \left(-\sqrt{\frac{\mathrm{i} \omega}{\alpha_{n}} z}\right)+G_{n}(z)$

where $A_{i}, B_{i}$ are coefficients, and

$G_{n}(z)=\left.\frac{1}{\sqrt{\mathrm{i} \omega} \varepsilon_{n}}\left\{\int \sinh \left[\sqrt{\frac{\mathrm{i} \omega}{\alpha_{n}}}(\xi-z)\right] w_{0 n}(\xi) \mathrm{d} \xi\right\}\right|_{\xi=z}$.

An analysis of the temperature field in the layered system can be done by the quadrupole method, an idea of which is described in Carslaw and Jaeger's fundamental textbook on heat transport [10]. The harmonic components of the temperature $\theta_{n}$ and heat flux $j_{n}$ in each layer are given by the formula

$$
\begin{aligned}
{\left[\begin{array}{c}
\theta_{n}(z) \\
j_{n}(z)
\end{array}\right]=} & {\left[\begin{array}{cc}
1 & 0 \\
0 & \sqrt{\mathrm{i} \omega} \varepsilon_{n}
\end{array}\right]\left\{\left[\begin{array}{cc}
\exp \left(\sqrt{\frac{\mathrm{i} \omega}{\alpha_{n}}} z\right) & \exp \left(-\sqrt{\frac{\mathrm{i} \omega}{\alpha_{n}}} z\right) \\
-\exp \left(\sqrt{\frac{\mathrm{i} \omega}{\alpha_{n}}} z\right) & \exp \left(-\sqrt{\frac{\mathrm{i} \omega}{\alpha_{n}}} z\right)
\end{array}\right]\right.} \\
& \left.\times\left[\begin{array}{c}
A_{n} \\
B_{n}
\end{array}\right]+\left[\begin{array}{c}
G_{n}(z) \\
-\frac{1}{\sqrt{\mathrm{i} \omega} \varepsilon_{n}} \frac{\partial G_{n}(z)}{\partial z}
\end{array}\right]\right\}
\end{aligned}
$$

Because the air layers above and under the sample are assumed to be semi-infinite and non-absorbing, the last equation for these layers is

$$
\begin{aligned}
& {\left[\begin{array}{c}
\theta_{0}(z) \\
j_{0}(z)
\end{array}\right]} \\
& \quad=\left[\begin{array}{cc}
1 & 0 \\
0 & \sqrt{\mathrm{i} \omega} \varepsilon_{\mathrm{a}}
\end{array}\right]\left[\begin{array}{cc}
\exp \left(\sqrt{\frac{\mathrm{i} \omega}{\alpha_{\mathrm{a}}}} z\right) & \exp \left(-\sqrt{\frac{\mathrm{i} \omega}{\alpha_{\mathrm{a}}}} z\right) \\
-\exp \left(\sqrt{\frac{\mathrm{i} \omega}{\alpha_{\mathrm{a}}}} z\right) & \exp \left(-\sqrt{\frac{\mathrm{i} \omega}{\alpha_{\mathrm{a}}}} z\right)
\end{array}\right]\left[\begin{array}{c}
A_{0} \\
0
\end{array}\right],
\end{aligned}
$$

for the air above the sample and

$$
\begin{aligned}
{\left[\begin{array}{c}
\theta_{N+1}(z) \\
j_{N+1}(z)
\end{array}\right]=} & {\left[\begin{array}{cc}
1 & 0 \\
0 & \sqrt{\mathrm{i} \omega} \varepsilon_{\mathrm{a}}
\end{array}\right] } \\
& \times\left[\begin{array}{cc}
\exp \left(\sqrt{\frac{\mathrm{i} \omega}{\alpha_{\mathrm{a}}}} z\right) & \exp \left(-\sqrt{\frac{\mathrm{i} \omega}{\alpha_{\mathrm{a}}}} z\right) \\
-\exp \left(\sqrt{\frac{\mathrm{i} \omega}{\alpha_{\mathrm{a}}}} z\right) & \exp \left(-\sqrt{\frac{\mathrm{i} \omega}{\alpha_{\mathrm{a}}}} z\right)
\end{array}\right] \\
& \times\left[\begin{array}{c}
0 \\
B_{N+1}
\end{array}\right],
\end{aligned}
$$

for the air below it, where $\alpha_{\mathrm{a}}$ is the thermal diffusivity and $\varepsilon_{\mathrm{a}}$ is the thermal effusivity of air. Boundary conditions at the $z_{n}=\sum_{k=1}^{n} d_{k}$ interface can be written as

$\left[\begin{array}{c}\theta_{n}\left(z_{n}\right) \\ j_{n}\left(z_{n}\right)\end{array}\right]=\left[\begin{array}{c}\theta_{n+1}\left(z_{n}\right) \\ j_{n+1}\left(z_{n}\right)\end{array}\right]$

Solving the system of (9), the temperature field in the sample and its surrounding can be calculated. In this work, homogeneous, single-layer samples, and non-homogeneous, twoand three-layer samples were considered in analysis of experimental data.

To analyze experimental data, the knowledge of $A_{0}$ and $B_{N+1}$ is sufficient. The temperature of the rear sample surface is given by

$\theta_{N}(D)=B_{N+1} \exp \left(-\sqrt{\frac{i \omega}{\alpha_{\mathrm{a}}}} D\right)$

where $D=\sum_{k=1}^{N} d_{k}$ is the thickness of the sample, while the temperature of the front surface is given by

$\theta_{0}(D)=A_{0}$

It should be noticed here that both $A_{0}$ and $B_{N+1}$ are complex functions of $\omega$, thicknesses and thermal parameters of the layers $d_{n}, \alpha_{n}, \varepsilon_{n}$, and distributions of heat sources $w_{0 n}(z)$. In the simplest approximation, the deflection of the probe beam in the second measuring method (geometry in Fig. 1b) can be assumed to be proportional to the temperature gradient in the air above the sample:

$S=\left.K \frac{\partial \theta_{0}}{\partial z}\right|_{z=h}=-K \sqrt{\frac{\mathrm{i} \omega}{\alpha_{\mathrm{a}}}} A_{0} \exp \left(-\sqrt{\frac{\mathrm{i} \omega}{\alpha_{\mathrm{a}}}} h\right)$,

where $h$ is the distance between the probe beam and the sample surface, and $K$ is a constant of proportionality [11]. 
To determine the thermal parameters of CEG samples, measured dependences of the phase on the square root of modulation frequency were fitted with dependences calculated using the presented model. It was assumed that the investigated samples are opaque, so heat sources arise only at the illuminated sample surface. The thermal diffusivity of air is taken as $0.2 \mathrm{~cm}^{2} \mathrm{~s}^{-1}$. In order to reduce the number of fitted parameters, the following combinations of parameters are used in numerical procedures: $d_{n} \alpha_{n}^{-1 / 2}, \varepsilon_{n} \varepsilon_{\mathrm{a}}^{-1}$, and $h \alpha_{\mathrm{a}}^{-1 / 2}$. The fitting procedures were written in MATLAB using its standard functions.

\section{Results and discussion}

The thermal diffusivity in the parallel direction was measured for all samples listed in Table 1 in the configuration shown in Fig. 1a. Exemplary results obtained for the sample GEP-22 fitted with the theoretical curve are shown in Fig. 3. The determined thermal diffusivity is $0.64 \mathrm{~cm}^{2} \mathrm{~s}^{-1}$ with an uncertainty of about $15 \%$. The fitting procedure returns also the ratio of the thermal effusivity of the sample to the one of the air. However, a sensitivity of the fitting procedure to this parameter is rather low and obtained values should be treated as rough estimation. Figure 4 shows the dependence of the parallel thermal diffusivity on the apparent density of CEG. Under the assumption that the specific heat of CEG is approximately the same as for graphite ( $c=850 \mathrm{~J} \mathrm{~kg}^{-1} \mathrm{~K}^{-1}[5]$ ), the parallel thermal conductivities were also calculated using the relation $\kappa=\alpha \rho c$. Results are shown in Fig. 4. The parallel thermal diffusivity reaches a maximum for the apparent density of about $50 \mathrm{~kg} \mathrm{~m}^{-3}$ and then monotonically decreases with increasing density. The

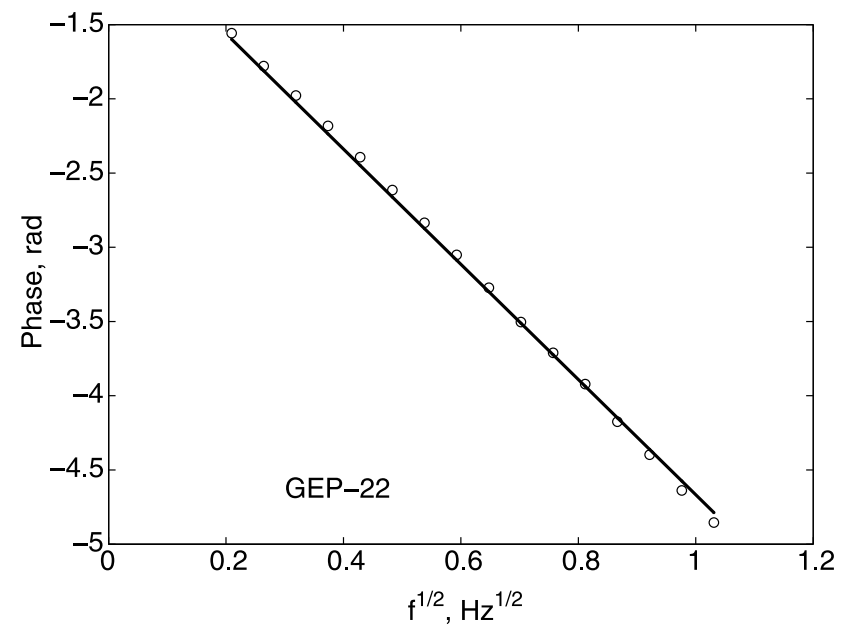

Fig. 3 Measured dependence of the phase of the temperature disturbance at the rear surface as a function of square root of the modulation frequency (circles). Results were obtained for the sample GEP-22. The fitted theoretical dependence is also shown (solid line) parallel thermal conductivity increases with growing density for low-density samples and probably has a plateau for densities higher than $200 \mathrm{~kg} \mathrm{~m}^{-3}$. Such behavior is consistent with results published by other authors.

In the next step, the anisotropy of the thermal properties of CEG was examined. Plates with surfaces parallel to the compressing direction were cut out from selected samples and perpendicular thermal diffusivities were measured. As in the previous case, thermal conductivities were also calculated. The results, together with those obtained for the parallel direction for the same samples, are shown in Figs. 5 and 6 . By contrast with the parallel thermal diffusivity, the perpendicular one increases with growing apparent density of CEG. The same dependence is observed for the perpendicular thermal conductivity. The apparent density in-

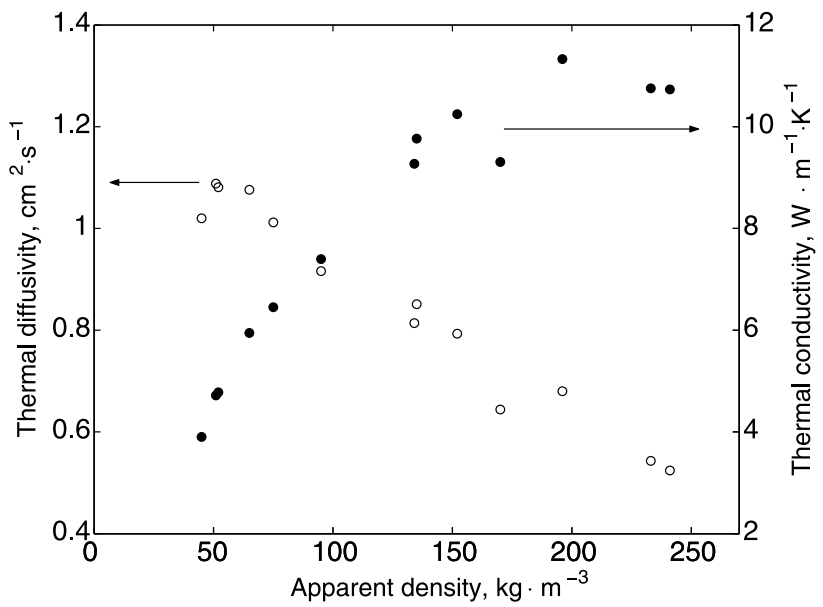

Fig. 4 The thermal diffusivity (empty circles) and the thermal conductivity (filled circles) of CEG in the parallel direction as a function of the apparent density

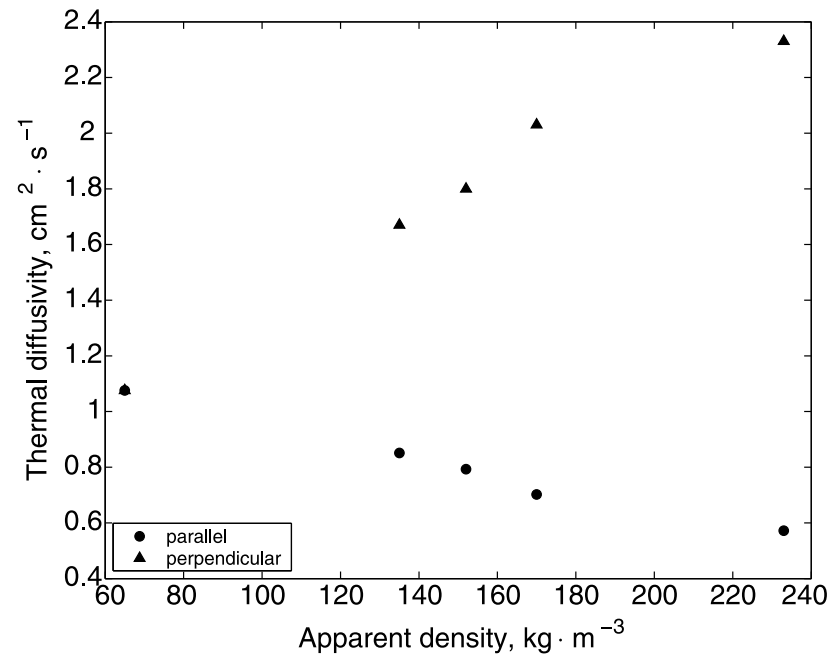

Fig. 5 The thermal diffusivity dependence on the apparent density of CEG in directions parallel (circles) and perpendicular (triangles) to the direction of compression 
creases, and the differences between the thermal conductivities and thermal diffusivities in both directions increase. It can also be noticed that these differences disappear for samples of very low density. An explanation of this effect was proposed by Celzard et al. [4]. In low-density CEG the graphene planes are randomly oriented in space and the material is isotropic. During preparation of more dense samples, a pressing stress is higher and graphene planes arrange predominantly perpendicularly to the compressing direction. As a result, the thermal conductivity in the perpendicular direction occurs mainly along graphene planes and this mechanism of heat transport is very effective. In natural and synthetic crystalline graphite the thermal conductivity along these planes can be more than two orders

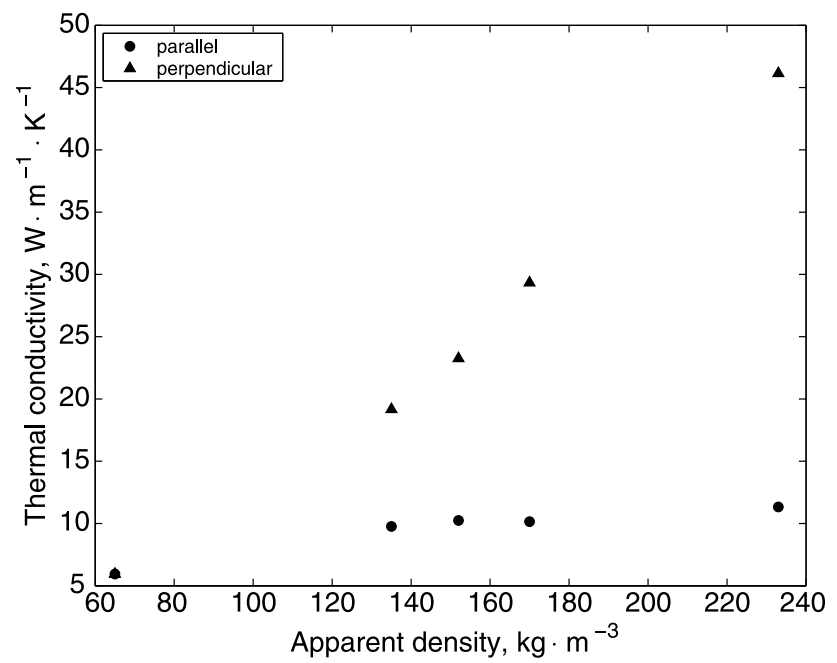

Fig. 6 The thermal conductivity dependence on the apparent density of CEG in directions parallel (circles) and perpendicular (triangles) to the direction of compression

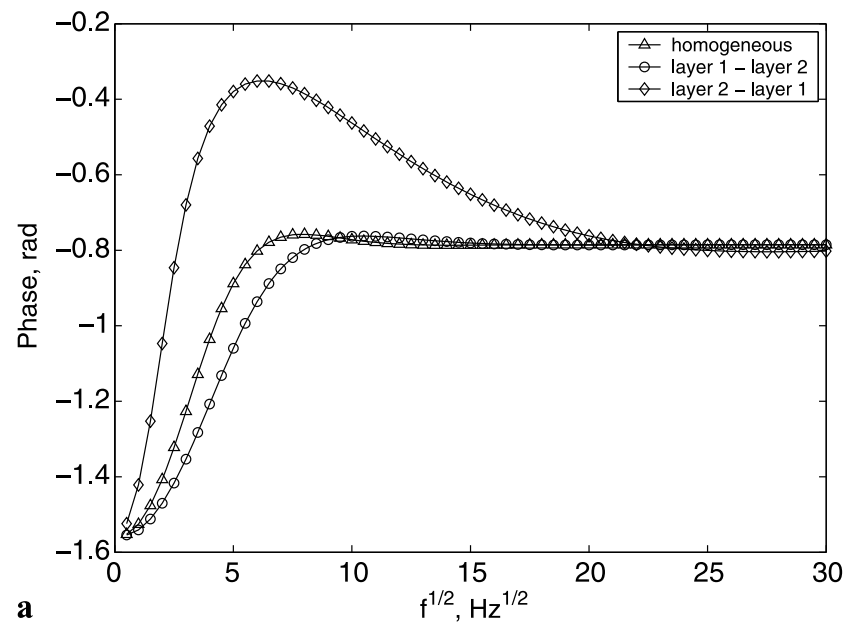

Fig. 7 Calculated phase of temperature disturbance at front (a) and rear (b) surfaces of different samples. Analysis was carried out for homogeneous (line with triangles) and two-layered samples (line with of magnitude higher than in the direction perpendicular to them [12]. The measured thermal anisotropy (the ratio of perpendicular and parallel thermal conductivities) for typical graphite laminate materials is about 50 [13]. Changes in the thermal conductivity with growing apparent density can be explained by two effects. The first one is connected with orientation of graphene planes. As a result, the inner structure of a material becomes similar to the one of crystalline graphite. This process manifests in growing anisotropy of the thermal conductivity and diffusivity. Simultaneously, the material is more densely packed. Air voids between graphite flakes shrink. The heat transfer is done by a combination of conduction through graphite flakes and air voids. Such a model was proposed in Ref. [14]. The voids are bad thermal conductors and lower the effective thermal conductivity of the sample. Mean void dimensions go down with growing sample density and their contribution to the effective thermal conductivity also decreases. Therefore, an increase of the thermal conductivity with growing apparent density of the material is observed for both analyzed directions (Fig. 6).

As is mentioned in the introduction, measurements carried out in the geometry shown in Fig. 1a give averaged thermal parameters of the sample. On the other hand, in the review paper [5] a suggestion that CEG samples produced by compacting of EG can be non-homogeneous in the direction of compaction can be found. To check this suggestion, a few samples were cut into slices and the parallel thermal diffusivity of these slices was determined. In the case of the sample GEP-23, the thermal diffusivity of the central part was slightly lower than for outer parts, but the difference was comparable with the uncertainty of the measurements. However, this result encouraged us to carry out experiments in the geometry shown in Fig. 1b, whose results should reveal possible non-homogeneity of samples. In Fig. 7, results

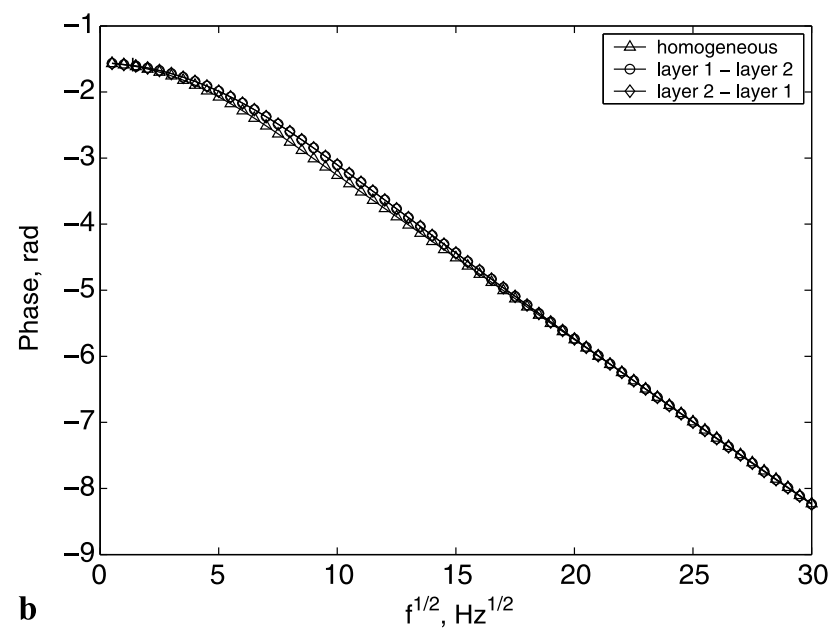

circles and line with diamonds). The layered samples differ in the order of the layers 


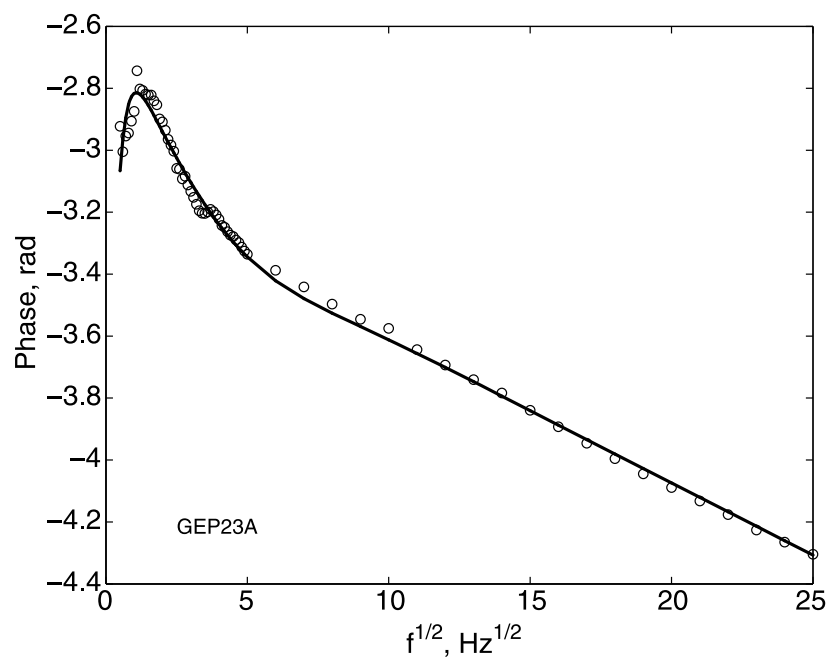

Fig. 8 The dependence of the phase of the deflection signal on frequency square root. The measurement was carried out for the 4.7-mm-thick outer part of the sample GEP-23. Experimental points were fitted by a theoretical curve calculated from a two-layer model

of numerical calculations of the phase of the temperature disturbance at front and rear sample surfaces are presented. These calculations were performed for three samples: a homogeneous one, and two samples consisting of two layers of different thermal properties. The layered samples differ in the order of the layers. The assumed parameters of the samples were: $d / \alpha^{1 / 2}=0.14 \mathrm{~s}^{1 / 2}, \varepsilon / \varepsilon_{\mathrm{a}}=1000$ for the homogeneous sample, $d_{1} / \alpha_{1}^{1 / 2}=0.10 \mathrm{~s}^{1 / 2}, \varepsilon_{1} / \varepsilon_{\mathrm{a}}=1000$ for layer 1 , and $d_{2} / \alpha_{2}^{1 / 2}=0.04 \mathrm{~s}^{1 / 2}, \varepsilon_{2} / \varepsilon_{\mathrm{a}}=200$ for layer 2 . The parameters were chosen in such a way that the equation

$$
\frac{d}{\sqrt{\alpha}}=\frac{d_{1}}{\sqrt{\alpha_{1}}}+\frac{d_{2}}{\sqrt{\alpha_{2}}}
$$

is fulfilled. Calculated dependences of the phase of the temperature disturbance on the front surface are different for different samples (Fig. 7a), while these dependences calculated for the rear surface are almost identical (Fig. 7b). It means that possible non-homogeneity of CEG samples should be visible in front-side measurement results.

An analysis of experimental dependences of the phase of the mirage signal on modulation frequency square root obtained for different samples led to a conclusion that there are discrepancies between theoretical predictions based on the one-layer 1D model and experimental data. This effect is especially obvious for samples with relatively high apparent density. As an example, results of measurements carried out for the 4.7-mm-thick outer part of the sample GEP-23 are shown in Fig. 8. An attempt to fit this dependence with a curve-calculated 1D model for a homogeneous sample did not give a satisfactory effect. However, when the two-layer model had been adopted, very good agreement between experimental and theoretical dependences was achieved. Val- ues of fitted parameters were: $d_{1} / \alpha_{1}^{1 / 2}=0.19 \mathrm{~s}^{1 / 2}$ and $d_{2} / \alpha_{2}^{1 / 2}=0.48 \mathrm{~s}^{1 / 2}$. The effective thermal diffusivity of this sample was also measured by the transmission technique and is $0.49 \mathrm{~cm}^{2} \mathrm{~s}^{-1}$. So, $d / \alpha^{1 / 2}$ for it is $0.67 \mathrm{~s}^{1 / 2}$. The fitting procedure was written in such a way that parameters $d_{1} / \alpha_{1}^{1 / 2}$ and $d_{2} / \alpha_{2}^{1 / 2}$ have to fulfill (13). It lowers the number of fitted parameters and improves reliability of fitting. In a general case, the analysis of data obtained from front-detection measurement does not allow us to determine the thermal diffusivities and thicknesses of the layers. But, if additional information about the thickness or the thermal diffusivity of one layer is available, complete information about the layers can be retrieved. In the considered case, it is justified to assume that $\alpha_{2}$ is the same as the thermal diffusivity of the central part of the sample GEP-23, which was measured independently. The obtained value is $0.45 \mathrm{~cm}^{2} \mathrm{~s}^{-1}$. So, from $d_{2} / \alpha_{2}^{1 / 2}=0.48 \mathrm{~s}^{1 / 2}$ we have $d_{2}=3.25 \mathrm{~mm}$, and from the overall sample thickness one can calculate the thickness of the outer layer $d_{1}=1.45 \mathrm{~mm}$. Finally, the thermal diffusivity $\alpha_{1}$ is $0.58 \mathrm{~cm}^{2} \mathrm{~s}^{-1}$. These results should be treated as rough estimation, but they give a general idea about the sample structure.

The obtained agreement between measured dependences and theoretical predictions justifies that in both experimental geometries a simple 1D model of thermal wave propagation is sufficient for data analysis. A deviation from it could be expected at very low frequencies.

\section{Conclusions}

It was shown that continuous-wave photothermal techniques allow us to investigate thermal properties of relatively thick samples with an uncertainty of about $15 \%$. Transmission measurements give a value of the thermal diffusivity averaged along the propagation of the thermal wave. Such experiments are rather insensitive to the non-homogeneity of the sample. Measurements based on the interference of thermal waves reveal the inner structure of the sample. From this point of view, they provide more complete information about the sample. The main problem with interferometric measurements for thick samples is that the thermal wave travels twice through the sample. It requires experiments at very low frequencies.

The results obtained for CEG samples show that the thermal properties of CEG strongly depend on its apparent density. Samples of the density of about $50 \mathrm{~kg} \mathrm{~m}^{-3}$ are thermally isotropic. If the density increases, the thermal diffusivity and the thermal conductivity in the direction perpendicular to the compression direction rapidly increase, while the thermal diffusivity in the parallel direction slowly goes down. The parallel thermal conductivity grows $2-3$ times and reaches a plateau for apparent densities higher than 
about $200 \mathrm{~kg} \mathrm{~m}^{-3}$. For dense samples the anisotropy is high. In our experiments, the ratio of thermal conductivities in perpendicular and parallel directions was close to 5 at $200 \mathrm{~kg} \mathrm{~m}^{-3}$.

The interferometric measurements showed that CEG samples are non-homogeneous along the compression direction. This non-homogeneity is difficult to find by sectioning the sample. It can be caused by the fact that cutting partially destroyed the inner structure of the sample. But, as follows from photothermal measurements, the central part of the sample has lower parallel thermal diffusivity. At this point, it should be also noticed that the two-layer model used in this paper is only the simplest approximation of the real sample structure.

It can be stated as a final conclusion that the possibility of controlling the thermal properties of CEG and obtaining material with required thermal conductivity and thermal anisotropy make CEG very promising for application in heat-management systems.

Acknowledgement The authors acknowledge Silesian University of Technology for financial support under a statutory grant and Prof. Marta Krzesińska for providing CEG samples.

Open Access This article is distributed under the terms of the Creative Commons Attribution Noncommercial License which permits any noncommercial use, distribution, and reproduction in any medium, provided the original author(s) and source are credited.

\section{References}

1. A. Yoshida, Y. Hishiyama, M. Inagaki, Carbon 29, 1227 (1991)

2. M.S. Dresselhaus, G. Dresselhaus, Adv. Phys. 51, 1 (2002)

3. D.D.L. Chung, J. Mater. Sci. 22, 4190 (1987)

4. A. Celzard, J.F. Marêché, G. Furdin, Prog. Mater. Sci. 50, 93 (2005)

5. M. Bonnissel, L. Luo, D. Tondeur, Carbon 39, 2151 (2001)

6. L.L. Vovchenko, L.Yu. Matzui, A.A. Kulichenko, Inorg. Mater. 43, 597 (2007)

7. I.M. Afanasov, D.V. Savchenko, S.G. Ionov, D.A. Rusakov, A.N. Seleznev, V.V. Avdeev, Inorg. Mater. 45, 486 (2009)

8. W.J. Parker, R.J. Jenkins, C.P. Butler, C.L. Abbott, J. Appl. Phys. 32, 1679 (1961)

9. J. Bodzenta, A. Kaźmierczak-Bałata, J. Mazur, Cent. Eur. J. Phys. 8, 207 (2010)

10. H.S. Carslaw, J.C. Jaeger, Conduction of Heat in Solids (Clarendon, Oxford, 1980)

11. L.C. Aamodt, J.C. Murphy, J. Appl. Phys. 52, 4903 (1981)

12. G.A. Slack, Phys. Rev. 127, 694 (1962)

13. J. Norley, J.J.-W. Tzeng, G. Getz, J. Klug, B.F. Graftech, in IEEE Proc. 17th Semiconductor Thermal Measurement and Management Symposium (2001), p. 107

14. J.-P. Monchalin, L. Bertrand, G. Rousset, F. Lepoutre, J. Appl. Phys. 56, 190 (1984) 